\title{
A POLYGLANDULAR AUTOIMMUNE SYNDROME OF A DIFFERENT TYPE: A CASE REPORT
}

\author{
FLANNERY M.T.* \\ Department of Medicine, University of South Florida Morsani College of Medicine,12901 Bruce B. Downs Blvd., Tampa, Florida 33612. \\ ${ }^{*}$ Corresponding Author: Email- mflann5555@aol.com
}

Received: November 29, 2012; Accepted: January 15, 2013

\begin{abstract}
-
Introduction: I present a rare case of a previously unrecognized combination of affected organs systems in a middle aged Caucasian woman. Presentation: The patient presented with complications of celiac sprue including osteomalacia with fractures of the small bones of her feet and pernicious anemia. The patient subsequently presented with autoimmune hypoaldosteronism and secondary Addision's disease with deficient Adrenocorticotropin hormone. Finally, once the fore mentioned conditions were treated and stabilized, the patient developed uncomplicated Idiopathic Thrombocytopenia.
\end{abstract}

Conclusion: Patients suffering from any form of polyglandular autoimmune condition must be monitored closely and treated promptly to avoid increased morbidity and mortality.

Keywords- Celiac sprue, Common variable immunodeficiency, Pernicious anemia, Idiopathic Thrombocytopenia, Hypoaldoseronism, Secondary Addison's disease

Citation: Flannery M.T. (2013) A Polyglandular Autoimmune Syndrome of a Different Type: A Case Report. Medical Case Reports, ISSN: 0976 -8726 \& E-ISSN: 0976-8734, Volume 4, Issue 1, pp.-39-41.

Copyright: Copyright@2013 Flannery M.T. This is an open-access article distributed under the terms of the Creative Commons Attribution License, which permits unrestricted use, distribution and reproduction in any medium, provided the original author and source are credited.

\section{Introduction}

Patients may present with autoimmune conditions abruptly or, more commonly, slowly over time requiring increased vigilance on part of their physician. Though many combinations have been previously described and linked to genetic etiologies, most physicians deal with one problem at a time until there is a realization that a polyglandular process is ongoing. Obviously, one of the most feared autoimmune syndromes is adrenal insufficiency, since unrecognized, it can lead to death. A question that is commonly asked on board exams for primary care providers. We present a case of a patient with a variety of autoimmune diseases presenting over time and on occasion with a complete surprise.

\section{Case Presentation}

A 46 year old Caucasian female presented for primary care establishment with a history of pain in her feet for three months. He pain was throbbing, worsened with ambulation and did not refer. She had recently seen an Orthopedist 1-2 weeks previously who casted both lower extremities to a mid-point to the knee. The patient had experienced no trauma and was unsure why her casts were placed. She no medical records with her. On review of systems she had a 2 year history of recurrent urinary tract and sinopulmonary tract infections with a documented history of hypogammaglobulinemia (IgA and $(g G)$ treated with gammaglobulin infusions every two weeks. She also claimed to have various drug allergies or reactions to the fluoroquinolones and penicillin. Other medical problems included a seizure disorder, hepatitis $B$ and allergic rhinitis. There was no his- tory of diabetes mellitus or thyroid disease. She continued with normal menstrual periods and was a non-smoker. Her exam demonstrated normal vitals with a clean port in the left subclavicular region. She was under weight with a body mass index of 17 . She has no murmur, organomegaly or rash. Her casts were present bilaterally to the mid-tibia. Initial labs demonstrated mild leukopenia of $3.8 \times 10^{9} / \mathrm{L}$ and anemia with a hemoglobin of $82 \mathrm{~g} / \mathrm{L}$. The mean corpuscular volume was 81 with a normal platelet count. The transferrin saturation was $<10 \%$ with a ferritn $<10 \mathrm{pmol} / \mathrm{L}$. The reticulocyte count was $<1 \%$. Her electrolytes and creatinine were normal except for a calcium of $1.9 \mathrm{mmol} / \mathrm{L}$, an albumin of $28 \mathrm{~g} / \mathrm{L}$ and cholesterol of $3.24 \mathrm{mmol} / \mathrm{L}$. The protime was $15.9 \mathrm{~s}$ with a normal partial thromboplastin time. With borderline calcium levels her parathyroid hormone level was $197 \mathrm{ng} / \mathrm{L}$. Other than a high concentration her urinalysis was normal. Reports obtained later demonstrated small bone fractures of her feet bilaterally. A chest radiograph was normal. Subsequent to the labs, the patient was re-questioned about her bowel habits and she again denied diarrhea. However, she reported over 10 loose bowel movements daily which had been going on for a lengthy amount of time. She could not specify how much weight loss there may have been. Anti-tissue- transglutaminase, anti-endomysial, anti-reticulin and anti-gliadin autoantibodies were all negative. An esophagogastroduodenal study was done with biopsies of the proximal small bowel which demonstrated decrease villi, without organisms, potentially consistent with celiac disease. Her $25-\mathrm{OH}$ Vitamin D level was $30 \mathrm{nmol} / \mathrm{L}$. The patient was placed on vitamin $\mathrm{D}$ and $\mathrm{K}$ replacement, calcium and iron sup- 
plementation and a gluten free diet. This resulted in a decrease in her stool frequency and weight gain with near correction of her vitamin $D$, calcium and protime levels. Her iron deficiency minimally improved so she was treated with intravenous iron infusions which resulted in a marked reticulocytosis and increased her hemoglobin to $130 \mathrm{~g} / \mathrm{L}$.

For several months the patient was doing well and had her casts removed without residual bone pain. Her Dual-energy Xray absorptiometry (DEXA) scan demonstrated osteoporosis so a biphosphanate was added to her drug regimen. A repeat DEXA study, one year later, demonstrated a $3 \%$ improvement in the density of her hip and lumbar spine.Twelve months after her initial presentation she presented for follow-up and labs. Her exam and labs were normal except a sodium of $128 \mathrm{mmol} / \mathrm{L}$ and a potassium of $5.8 \mathrm{mmol} / \mathrm{L}$. The urinary sodium was $52 \mathrm{mmol} / \mathrm{L}$. Though her blood pressure and glucose were normal a cortisyn stimulation test was performed and was within normal limits. Subsequently, she underwent a dehydration stimulation test with pre and post-renin and aldosterone levels. Baseline levels were low and did not increase as anticipated. Her hemoglobinA1C was $5.9 \%$ and her creatinine remained in the low normal range. The patient was treated with fludrocortisone acetate with normalization of her sodium and potassium within 2 weeks. Five months later, the patient presented with abdominal pain and nausea and vomiting of one day duration. On presentation, she had a blood pressure of $78 / 42 \mathrm{mmHg}$ and a heart rate of 110 beats/ minute. Her mucous membranes were moist and her abdominal exam demonstrated minimal epigastric tenderness. Her stool was Hemmocult negative. An Accucheck was 59 which was confirmed by her serum glucose. A complete blood count showed a mild leukocytosis of $12.2 \times 10 \% / \mathrm{L}$ with $10 \%$ eosinophils. Her sodium and potassium were borderline low and high respectively with a low normal creatinine. She was treated with a 2 liter bolus of $5 \%$ dextrose and normal saline solution and $100 \mathrm{mg}$ of intravenous hydrocortisone. Prior to treatment, the patient underwent a draw of her serum ACTH level and a cortisyn stimulation test. The patient experienced immediate improvement without pain and normalization of her vitals to her baseline. A repeat Accucheck was 92 within 3 hours. The cortisyn stimulation values demonstrated a normal response, however, her adrenocorticotropic hormone (ACTH) level was less than $2 \mathrm{pg} / \mathrm{ml}$. A corticotropin stimulation test was performed 2 weeks later which demonstrated a blunted response to corticotropin releasing factor (CRF). A magnetic resonance imaging study of her head/pituitary with enhancement demonstrated no abnormalities. As this appeared to be acute pituitary dysfunction, she was continued on maintenance steroids daily with an emergency bracelet recommendation. The IGF1, TSH \& LH/FSH and prolactin levels were normal. Approximetely, 2 years later the patient developed isolated thrombocytopenia with a platelet count of $70 \times 10^{9}$. The patient had no bleeding and her count remained stable. She had no splenomegaly and her human immunodeficency viral testing was negative. Hepatitis $C$ and hepatitis $B$ surface antigen were negative. An antinuclear antibody was 1:640 with positive results for anti-parietal cell antibodies and anti-adrenal antibodies. AntidsDNA, anti-Smith and Anti-RNP were negative. The anti-platelet antibodies were negative, as well. Her B12 level was borderline at $202 \mathrm{pmol} / \mathrm{L}$ with an increase in her methylmalonate level requiring replacement. Over the years, she was desensitized to penicillin and fluoroquinolones so they could be used to minimize drug resistance. Her infection rate, pulmonary and urinary, decreased over time.

\section{Discussion}

Our patient clearly had a form of polyglandular autoimmune syndrome.Her syndrome did not fit into any of the described polyglandular disorders and included immunodeficiency, clinical celiac sprue, autoimmune hyporenin/hypoaldosteronism (type 4 renal tubular acidosis) with hypo-ACTH response (secondary Addison's disease), immune thrombocytopenia and pernicious anemia. When she presented she had a history of immunodeficiency with recurrent mucosal infections and undiagnosed celiac sprue. Though one could argue that she did not have celiac disease with negative antibodies; an inappropriate lack of generation of antibodies may be present due to her history of immunodeficiency. The bowel biopsy is non-specific but she did respond very well to a gluten free diet with weight gain, decreased stools and nutritional improvement. It is not unusual to remain iron deficient given its difficult absorption properties. Subsequently, she most likely developed focused adrenal and pituitary antibodies resulting in her hyporenin/hypoaldosteronism and inappropriately low ACTH levels with a blunted response to CRF. This, interestingly, mimicked acute primary Addison's disease. She responded very well to treatment with steroids and fludrocortisone, a gluten free diet and Vitamin B12 replacement injections. Since her platelet count remained stable, without bleeding, it was monitored with observation.

There have been many types of autoimmune polyendocrine syndromes the most common of which is type II which has a $20 \%$ association with type I diabetes mellitus along with Addison's disease and thyroiditis. Type I is rare, occurs in infancy and is associated with candidiasis, hypoparathyroidism and Addision's disease. There is also a very rare neonatal X-linked version associated with neonatal diabetes and malabsorption. The theory is that there is a shared antigen that can result in targeting of multiple organs[1-3].

Our patient's various conditions do not match up with traditional autoimmune types or named conditions. Certainly there was evidence that she had common variable immunodeficiency (CVID) with immunoglobulin deficiencies and poor responses to immunization per her treating rheumatologist. One may argue whether she had celiac sprue at all, however, classic antibodies may be absent in CVID [4,5]. Also, she responded well with decreased stools, weight gain and nutritional improvement of Vitamin K and D. She clearly had osteomalcia with secondary hyperparathyroidism. The pernicious anemia may be multifactorial due to Vitamin B12 malabsorption and anti-parietal cell antibodies. The most interesting presentation came when she presented with abdominal pain, nausea/ vomiting, hypotension, hypoglycemia and eosinophilia classic for adrenal insufficiency. Given her prior diagnosis of hyporenin/ hypoaldosteronism, one would expect this to be an extension of primary adrenal insufficiency. However, subsequent data obtained just before therapy demonstrated an appropriate adrenal response but, likely, an acute autoimmune deficiency of ACTH. Given a normal magnetic resonance study of the head \& pituitary and normal results of other axises of the pituitary gland, makes such an acute deficiency likely to be immune in causation. The lack of proper response to CRF confirmed an improper ACTH response. With the development of another probable autoimmune idiopathic thrombocytopenia (ITP) by exclusion, her overall syndrome appeared to be autoimmune in nature including: CVID, secondary adrenal insufficiency, celiac sprue with resultant pernicious anemia and osteomalacia with fractures, hypoaldosteronism and ITP. Though the most common cause of a hyporenin/hypoaldoserone state is diabetes 
mellitus followed by drugs, sarcoidosis or obstructive uropathy, our patient had none of these conditions [6,7]. Again, this pointed to a probable autoimmune cause for this condition. There has also been a large study demonstrating a positive association between ITP before or after the diagnosis of celiac sprue [8]. In addition to her lack of diabetes mellitus, as seen in many other autoimmune states, she never developed thyroiditis during our care of the patient. She ultimately died when she was admitted to another facility with adhesional bowel obstruction and underwent surgery without stress dose steroids.

\section{Conclusion}

In summary, our case demonstrates a polyglandular autoimmune syrdrome not previously described in the literature with celiac sprue, CVID, autoimmune hyporenin/hypoldosteronism, autoimmune ACTH deficiency, pernicious anemia and ITP. Recognition of this condition and watching out for other associated autoimmune states [1-3]. is critical to proper patient management. Our patient was able to be managed for many years offering her a better quality of life with decreased morbidity and mortality. All patients with potential life threatening deficiencies of the ACTH-Adrenal axis (Primary or Secondary Addison's disease) should be encouraged to wear life saving emergency bracelets.

\section{Consent}

The author could not obtain consent from the patient as she was deceased as noted in the last sentence of the discussion. No family members could be located. Due to the interesting nature of her case, I had discussed possibly submitting it for publication in the future and she expressed positive interest.

\section{Competing Interests}

The author declares that he has no competing interests.

\section{References}

[1] Eisenbarth G.S., Gottlieb P.A. (2004) New England Journal of Medicine, 350(20), 2068-79.

[2] Baker J.R. (1997) Journal of the American Medical Association, 278(22), 1931-37.

[3] Bottazzo G.F., Doniach D. (1981) Minerva Medica., 72(37), 2511-32.

[4] Kalha I., Sellin J.H. (2004) Current Gastroenterology Reports, 6 , 377-83.

[5] Rashtak S., Marietta E.V., Murray J.A. (2009) Expert Rev. Clin. Immunol., 5(5), 593-604.

[6] Tan S., Burton M. (1981) Arch. Intern. Med., 141(1), 30-3.

[7] Knochel J.P. (1979) Ann. Rev. Med., 30, 145-53.

[8] Olen O., Montgomery S.M., Elinder G., Ekbom A., Ludvigsson

J.F. (2008) Scand. J. Gastroenterol., 43(4), 416-22. 